\title{
Incidence of gastrointestinal stromal tumours is underestimated: Results of a nation-wide study
}

\author{
Wim G. Goettsch ${ }^{\text {a }}$, Steffan D. Bos ${ }^{\text {a,d }}$, Nancy Breekveldt-Postma ${ }^{\text {a }}$, Mariel Casparie ${ }^{\text {, }}$, \\ Ron M.C. Herings ${ }^{\text {a,b }}$, Pancras C.W. Hogendoorn ${ }^{\mathrm{d}, *}$ \\ ${ }^{a}$ PHARMO Institute, Utrecht, The Netherlands \\ ${ }^{\mathrm{b}}$ Department of Pharmacoepidemiology and Therapy, UIPS, Utrecht University, Utrecht, The Netherlands \\ ${ }^{\mathrm{c}}$ Foundation PALGA, Utrecht, The Netherlands \\ ${ }^{\mathrm{d}}$ Department of Pathology, Leiden University Medical Center, Building 1, L1Q, P.O. Box 9600, 2300 RC Leiden, The Netherlands
}

Received 4 August 2005; received in revised form 13 September 2005; accepted 13 September 2005

Available online 15 November 2005

\begin{abstract}
Gastrointestinal stromal tumours (GIST) are identified by their specific morphology added with immunohistochemical staining with anti-CD117, the phenotypic marker for GIST in the majority of cases. In this study the reported incidence of GIST and GISTlike tumours before and after the routine availability of the specific diagnostic marker CD117 antigen was investigated. All patients with GIST or GIST-like tumours were selected from PALGA, a nation-wide network and registry of histo- and cytopathology in the Netherlands, to calculate the incidence in 1995 and longitudinally over time between 1998 and 2003. Pathological reports were retrieved to assess the type of immunostaining used and to assess the risk category for malignant behaviour according to the recently published consensus criteria. The annual incidence of GIST as distilled from the national pathology registry increased from 2.1 per million inhabitants in 1995 to 12.7 per million inhabitants in 2003. The incidence of GIST-like tumours decreased from 17.6 per million inhabitants in 1995 to 12.7 per million inhabitants in 2003. The incidences were stable from 2000 onwards. Additional analysis in 2003 indicated that more than $90 \%$ of the GIST tested was CD117 positive, compared to only 4\% of the GIST-like tumours. Almost $50 \%$ of the GIST was considered to be at high-risk for malignant behaviour, according to the consensus criteria. The increased incidence of GIST 1995-2003 is related to increased understanding of GIST pathobiology and the routine availability of the diagnostic immunohistochemical antibody directed against the CD117 antigen.
\end{abstract}

(C) 2005 Elsevier Ltd. All rights reserved.

Keywords: Gastrointestinal stromal tumours; Underreporting; Incidence; CD117 antigen; Malignant behaviour; GIST

\section{Introduction}

Gastrointestinal stromal tumours (GIST) are the most common mesenchymal tumours of the gastrointestinal tract $[1,2]$. These tumours can be lethal; five-year survival ranges between $35 \%$ and $65 \%$ and depends on tumour size, mitotic index and location.[3] The identification of GIST has become important since specific,

\footnotetext{
* Corresponding author. Tel.: +31 71 5269111; fax: +31 715142588.

E-mail address: p.c.w.hogendoorn@lumc.nl (P.C.W. Hogendoorn).
}

pathogenesis-targeted treatment with Kit tyrosine kinase inhibitor, imatinib mesylate (Glivec ${ }^{\circledR}$ ) has become available, which has demonstrated promising clinical results [4-6]. Before the introduction of imatinib, patients with inoperable GIST had limited therapeutic options, with a low response rate (less than $10 \%$ ) to conventional chemotherapy $[4,7]$.

Information on the true incidence and prevalence of GIST is however, scarce, due to previous lack of well-defined pathologic criteria. Recent insights into the origin and pathogenesis of GIST revealed that pathologic acti- 
vation of Kit signal transduction is a central event of GIST pathogenesis, which usually leads to expression of Kit protein (CD117) [1,2]. This resulted in the development of a reliable phenotypic marker for GIST (CD117 antigen) and a major reappraisal of the classification of GIST and GIST-like tumours over the past 34 years $[4,8]$. Recent data from Sweden however may indicate that, on basis of CD117 testing, the annual incidence of GIST, has been largely underestimated [9].

The aim of the present study was to investigate the reported incidence of GIST and GIST-like tumours before and after the availability of the specific diagnostic marker CD117 antigen in a nation-wide study, making use of the unique situation of a national pathology registry covering $100 \%$ of pathology labs in the country. In addition, the metastatic risk index of reported GIST was assessed.

\section{Materials and methods}

\subsection{The PALGA registry}

Data for this study were obtained from PALGA, a nation-wide network and registry of histo- and cytopathology, which contains standardised abstracts of all 16 million inhabitants of The Netherlands since 1990. The abstracts contain amongst others encrypted patient identification, demographic data and a summary of the pathology report coded in accord with SNOMED terminology. The registry is both used for daily patient-care as for national and international scientific research.

\subsection{Retrieval of patients with GIST and GIST-like tumours}

From the PALGA registry all GIST and non-extremity GIST-like tumours (leiomyoma, leiomyosarcoma, liposarcomas, desmoid type fibromatosis, schwannoma and leiomyoblastoma) were selected in 1995 and between 1998 and 2003 [10]. The final selection of the cases was based on the coding of GIST and additional information from the conclusion; if a patient was coded as GIST-like tumour but referred to as a GIST in the conclusion the patient was classified as a GIST patient. Patients were included in the study at the time of their first diagnosis of GIST. For all cases of GIST and GIST-like tumours in 2003 the pathological reports were derived from the departments of pathology in order to assess additional information on the type of immunoassaying, tumour size and mitotic rate.

\subsection{Morphologic analyses}

For all cases of GIST and GIST-like tumours in 2003, the immunohistochemical staining results
(CD117, CD34, desmin, smooth muscle cell actin, vimentin) used to identify the tumour were assessed. The size of the tumour and its mitotic rate per 50 high power fields (HPF) were determined and these criteria were used for the risk assessment of GIST according to Fletcher [11]. This scheme includes four tumour risk groups, including a very-low-risk group $(<2 \mathrm{~cm}$ and $<5$ mitoses/50 HPF), a low-risk group $(2-5 \mathrm{~cm}$ and $<5$ mitoses $/ 50 \mathrm{HPF})$, an intermediate-risk group $(<5 \mathrm{~cm}$ and $6-10$ mitoses $/ 50 \mathrm{HPF}$ or $5-10 \mathrm{~cm}$ and $<5$ mitoses $/ 50 \mathrm{HPF}$ ), and a high-risk group $(>5 \mathrm{~cm}$ and $>5$ mitoses $/ 50 \mathrm{HPF}$ or $>10 \mathrm{~cm}$ regardless of mitotic activity or $>10$ mitoses/50 HPF regardless of the tumour size).

\subsection{Statistical methods}

The incidence of GIST and GIST-like tumours was calculated from the number of patients with a GIST or GIST-like tumour according to the PALGA data divided by the number of inhabitants in The Netherlands for 1995 and between 1998 and 2003. The incidence was standardised for differences in age (five-year classes) and gender in the Dutch population in time by using the Dutch population in 2003 as the standard population.

\section{Results}

\subsection{Incidence of GIST and GIST-like tumours}

The total annual incidence of GIST or GIST-like tumours increased from 20.8 per million inhabitants in 1995 to 25.4 per million inhabitants in 2003 (Table 1 and Fig. 1). Simultaneously, the annual incidence of GIST increased from 2.1 to 12.7 per million inhabitants whereas the annual incidence of GIST-like tumours, mostly leiomyomas and leiomyosarcomas, decreased from 18.7 to 12.7 per million inhabitants.

Data from the abstracts of the pathology reports showed that in 1995 about $72 \%$ of GIST was originally coded as non-GIST compared to only 1\% in 2003 (data not shown).

\subsection{Immunohistochemical properties of GIST and GIST- like tumours}

For 325 out of $411(79 \%)$ patients diagnosed with GIST in 2003, pathological reports were obtained. In 2003, staining with anti-CD117 was performed for $87 \%$ of the GIST and $93 \%$ of the tested GIST were CD117 positive (Table 2). Only $8 \%$ of the GIST-like tumours tested for CD117 were reported CD117 positive. About $74 \%$ of GIST were tested for the haematopoietic progenitor cell antigen CD34 and $83 \%$ was CD34 


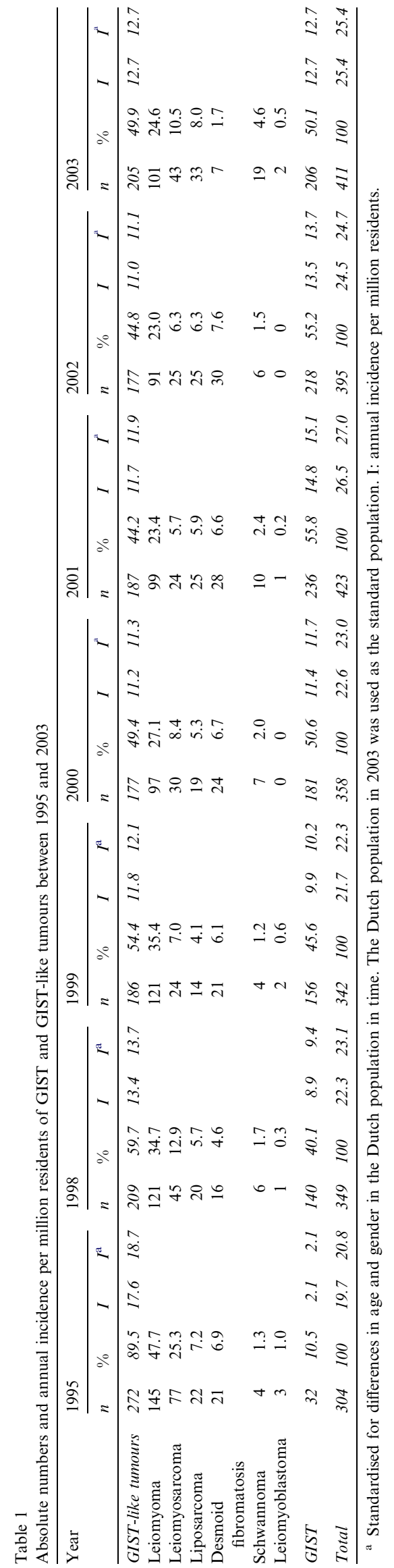

positive. A minority (40\%) of the GIST were tested for vimentin, and like most mesenchymal tumours, the majority of the GIST tested ( $89 \%$ ) were positive. In case of GIST-like tumours, $94 \%$ of the tested tumours were vimentin positive. Negative cases were most likely due to fixation artefacts potentially leading to false negative results for other markers as well. This percentage of vimentin negative cases was comparable to those encountered in the recent multi-institute phase III trials (manuscript in preparation).

The muscle cell markers desmin, smooth muscle cell marker (SMA) and the neural cell marker S100 were negative for most GIST tumours tested.

\subsection{Defining risk of aggressive behaviour of GIST}

For 124 of 159 patients (78\%) sufficient information on tumour size and mitotic rate was available from the pathological reports derived from the departments of pathology to define the risk of aggressive behaviour of the GIST in 2003 (Table 3). The majority of GIST had a low mitotic rate $(<1$ per $10 \mathrm{HPF})$, whereas only a low percentage of GIST ( $8 \%$ ) had a small tumour size $(<2 \mathrm{~cm})$. The majority $(45 \%)$ of the GIST were assessed as being at high-risk for malignant behaviour, followed by $31 \%$ of the GIST being at intermediate-risk.

\section{Discussion}

The present study shows that the reported annual incidence of GIST increased from 2.1 per million inhabitants in 1995 to 12.7 per million inhabitants in 2003 (206 patients). As a result, the incidence of GIST-like tumours decreased from 18.7 per million inhabitants in 1995 to 12.7 per million inhabitants in 2003 . Our results also showed that almost $45 \%$ of GIST were considered to be at high-risk for malignant behaviour in 2003 .

The increased incidence of GIST may be explained by an improved understanding of the pathobiology of GIST and the central role of activating Kit mutations in the pathogenesis of GIST, which led to the recognition of the new and reliable phenotypic marker CD117 antigen [12-14]. The reported incidence of GIST increased from 1998 onwards, before the general introduction of CD117 immunostaining on a regular basis in daily practice in 2001 . This may be related to an increased knowledge of the pathohistology of GIST. Tumours coded as GIST-like tumours in the registry were commonly characterised at the conclusions report as GIST by the pathologist exemplifying the ongoing debate on the characterisation of GIST. The true incidence of GIST is probably even slightly higher, including asymptomatic GIST and small and clinically insignificant GIST, which were not biopsied or resected at the time interval studied. 


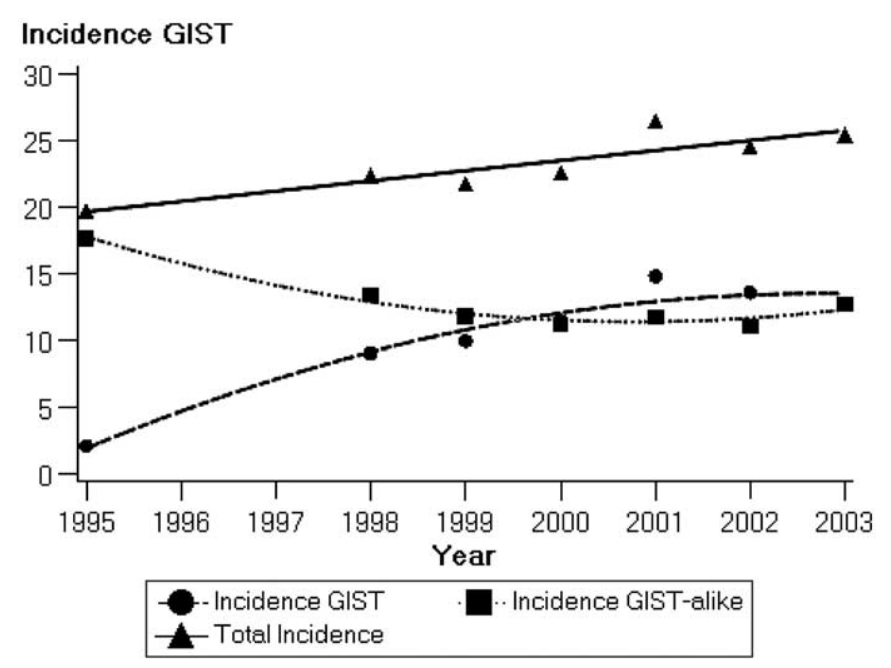

Fig. 1. Incidence of GIST and GIST-like tumours per one million residents from 1995 to 2003 , using the Dutch population in 2003 as the standard population.

Table 2

Immunohistochemical properties of GIST and GIST-like tumours in 2003

\begin{tabular}{|c|c|c|c|c|}
\hline \multirow[t]{2}{*}{ Patients } & \multicolumn{2}{|c|}{$\begin{array}{l}\text { GIST-like } \\
\text { tumours }^{\text {a }}\end{array}$} & \multicolumn{2}{|c|}{ GIST $^{\mathrm{a}}$} \\
\hline & $n$ & $\%$ & $n$ & $\%$ \\
\hline Total & 166 & 100 & 159 & 100 \\
\hline CD117 staining & 76 & 45.8 & 138 & 86.8 \\
\hline Positive & 6 & 3.6 & 129 & 81.1 \\
\hline Negative & 70 & 42.2 & 9 & 5.7 \\
\hline Unknown & 90 & 54.2 & 21 & 13.2 \\
\hline CD34 staining & 67 & 40.4 & 117 & 73.6 \\
\hline Positive & 10 & 6.0 & 97 & 61.0 \\
\hline Negative & 57 & 34.3 & 20 & 12.6 \\
\hline Unknown & 99 & 59.6 & 42 & 26.4 \\
\hline Desmin staining & 82 & 49.4 & 100 & 62.9 \\
\hline Positive & 59 & 35.5 & 13 & 8.2 \\
\hline Negative & 23 & 13.9 & 87 & 54.7 \\
\hline Unknown & 84 & 50.6 & 59 & 37.1 \\
\hline SMA staining & 98 & 59.0 & 112 & 70.4 \\
\hline Positive & 78 & 47.0 & 34 & 21.4 \\
\hline Negative & 20 & 12.1 & 78 & 49.1 \\
\hline Unknown & 68 & 41.0 & 47 & 29.6 \\
\hline S100 staining & 69 & 41.6 & 101 & 63.5 \\
\hline Positive & 20 & 12.1 & 13 & 8.2 \\
\hline Negative & 49 & 29.5 & 88 & 55.4 \\
\hline Unknown & 97 & 58.4 & 58 & 36.5 \\
\hline Vimentin staining & 49 & 29.5 & 64 & 40.3 \\
\hline Positive & 46 & 27.7 & 57 & 35.9 \\
\hline Negative & 3 & 1.8 & 7 & 4.4 \\
\hline Unknown & 117 & 70.5 & 95 & 59.8 \\
\hline
\end{tabular}

${ }^{\text {a }}$ From 325 of the 411 patients with GIST or GIST-like tumour pathological reports were obtained from the laboratory.

Few population-based data on the true incidence of GIST are available and this report describes the results of the first nation-wide study on the incidence of GIST
Table 3

Mitotic rate per $10 \mathrm{HPF}$ and tumour size of GIST-like tumours and GISTs in 2003

\begin{tabular}{|c|c|c|}
\hline \multirow[t]{2}{*}{ Patients } & \multicolumn{2}{|c|}{ GIST } \\
\hline & $n$ & $\%$ \\
\hline Total & 159 & 100 \\
\hline $\begin{array}{l}\text { Mitotic rate } \\
<1 \text { Per } 10 \mathrm{HPF} \\
1-2 \text { Per } 10 \mathrm{HPF} \\
>2 \text { Per } 10 \mathrm{HPF}\end{array}$ & $\begin{array}{l}96 \\
28 \\
35\end{array}$ & $\begin{array}{l}60.4 \\
17.6 \\
22.0\end{array}$ \\
\hline $\begin{array}{l}\text { Size } \\
<2 \mathrm{~cm} \\
2-5 \mathrm{~cm} \\
5-10 \mathrm{~cm} \\
\text { More than } 10 \mathrm{~cm} \\
\text { Biopt }^{\mathrm{a}}\end{array}$ & $\begin{array}{l}13 \\
43 \\
35 \\
25 \\
43\end{array}$ & $\begin{array}{r}8.2 \\
27.0 \\
22.0 \\
15.7 \\
27.0\end{array}$ \\
\hline $\begin{array}{l}\text { Risk assessment }^{\mathrm{b}} \\
\text { Very-low-risk } \\
\text { Low-risk } \\
\text { Intermediate-risk } \\
\text { High-risk }\end{array}$ & $\begin{array}{l}12 \\
18 \\
38 \\
56\end{array}$ & $\begin{array}{r}9.7 \\
14.5 \\
30.6 \\
45.2\end{array}$ \\
\hline
\end{tabular}

${ }^{a} \mathrm{~A}$ biopt was taken and information on tumour size was unavailable.

${ }^{\mathrm{b}}$ Excluding patients with a biopt except biopsies with a mitotic rate $>10 / 50 \mathrm{HPF}$ which are classified as high-risk independent of tumour size $(N=124)$.

and GIST-like tumours based on pathology registration. The recent annual incidence of GIST in the Netherlands is in range with the estimated incidence recently reported for Sweden (14 per million inhabitants) [9]. In this study, records of patients in a part of Sweden over the period of 1983-2000 were re-evaluated and 288 of those patients were diagnosed as having GIST tumours. According to their data, the annual incidence of GIST did not differ significantly over time. In our study no re-evaluation took place and the results 
represent data from the entire Dutch population from 1995 to 2003. The increase in the observed incidence of GIST until 2000 reflects the increased knowledge on the diagnostic pathology criteria of GIST. After the introduction of immunohistochemical staining against CD117, the incidence of GIST seems to be rather stable.

Our additional analysis in 2003 indicate that more than $90 \%$ of the GIST tested were CD117 positive which is in correspondence to previously reported percentage $(85-95 \%)$ of GIST that express Kit $[4,8]$. Our study also shows that only $4 \%$ of the GIST-like tumours were reported CD117 positive. This most probably reflects the false positive staining rate outside the setting of reference laboratories. Additional results on CD34 (93\%) positivity and absence for desmin (87\%) show that the characterisation of the GIST has been well established in the Netherlands and these tests are commonly used by pathologists as additional information to differentiate between GIST and GIST-like tumours.

The present study shows that about $45 \%$ of the GIST were assessed as being at high-risk for malignant behaviour, based on mitotic rate and tumour size [11]. This was similar to the percentage reported for Sweden [9]. This study showed that the median overall survival was 14 years or higher for patients with an intermediate or lower-risk of malignant behaviour, whereas the median overall survival was only $1.5-3.4$ years for patients with a high-risk for malignant behaviour. The estimated number of persons at high-risk for malignant behaviour was about 5-6 persons per million per year in The Netherlands. The true number may be higher, because the metastatic risk could not be assessed in part of the patients with a biopsy. Recently the consensus meeting for the management of GIST was held and its results published [15]. Information on the metastatic risk of GIST is important for its clinical management. Clinical trials showed high response rates (about $45-65 \%$ partial response and $20-30 \%$ stable disease) with imatinib in the advanced and metastatic setting [4-6]. At present, the role of imitanib in the adjuvant setting is under investigation $[4,16]$.

\section{Conflict of interest statement}

None declared.

\section{Acknowledgements}

The contribution to this study of the Dutch national pathology database 'Stichting PALGA' and the additional support of the individual pathological laborato- ries are fully acknowledged. Prof Hogendoorn acts in the (inter)national advisory board of Novartis-Pharma; this study was supported by an unrestrictional research grant of Novartis-Pharma, Arnhem, The Netherlands.

\section{References}

1. Nishida T, Hirota S. Biological and clinical review of stromal tumors in the gastrointestinal tract. Histol Histopathol 2000, 15(4), 1293-1301.

2. Miettinen M, Lasota J. Gastrointestinal stromal tumors definition, clinical, histological, immunohistochemical, and molecular genetic features and differential diagnosis. Virchows Arch 2001, 438(1), 1-12.

3. Roberts PJ, Eisenberg B. Clinical presentation of gastrointestinal stromal tumors and treatment of operable disease. Eur J Cancer 2002, 38(Suppl. 5), S37-S38.

4. D'Amato G, Steinert DM, McAuliffe JC, et al. Update on the biology and therapy of gastrointestinal stromal tumors. Cancer Control 2005, 12(1), 44-56.

5. Verweij J, Casali PG, Zalcberg J, et al. Progression-free survival in gastrointestinal stromal tumours with high-dose imatinib: randomised trial. Lancet 2004, 364(9440), 1127-1134

6. Demetri GD, von Mehren M, Blanke CD, et al. Efficacy and safety of imatinib mesylate in advanced gastrointestinal stromal tumors. New Engl J Med 2002, 347(7), 472-480.

7. Dematteo RP, Heinrich MC, El-Rifai WM, et al. Clinical management of gastrointestinal stromal tumors: before and after STI-571. Hum Pathol 2002, 33(5), 466-477.

8. Miettinen M, Majidi M, Lasota J. Pathology and diagnostic criteria of gastrointestinal stromal tumors (GISTs): a review. Eur $J$ Cancer 2002, 38(Suppl. 5), S39-S51.

9. Nilsson B, Bumming P, Meis-Kindblom JM, et al. Gastrointestinal stromal tumors: the incidence, prevalence, clinical course, and prognostication in the preimatinib mesylate era - a population-based study in western Sweden. Cancer 2005, 103(4), $821-829$.

10. Graadt van Roggen JF, van Velthuysen ML, Hogendoorn PC. The histopathological differential diagnosis of gastrointestinal stromal tumours. J Clin Pathol 2001, 54(2), 96-102.

11. Fletcher CD, Berman JJ, Corless C, et al. Diagnosis of gastrointestinal stromal tumors: a consensus approach. Hum Pathol 2002, 33(5), 459-465.

12. Sarlomo-Rikala M, Kovatich AJ, Barusevicius A, et al. CD117: a sensitive marker for gastrointestinal stromal tumors that is more specific than CD34. Mod Pathol 1998, 11(8), 728-734.

13. Kindblom LG, Remotti HE, Aldenborg F, et al. Gastrointestinal pacemaker cell tumor (GIPACT): gastrointestinal stromal tumors show phenotypic characteristics of the interstitial cells of Cajal. Am J Pathol 1998, 152(5), 1259-1269.

14. Arber DA, Tamayo R, Weiss LM. Paraffin section detection of the c-kit gene product (CD117) in human tissues: value in the diagnosis of mast cell disorders. Hum Pathol 1998, 29(5), 498-504.

15. Blay J-Y, Bonvalot $\mathrm{S}$, Casali $\mathrm{P}$, et al. Consensus meeting for the management of gastrointestinal stromal tumors * Report of the GIST Consensus Conference of 20-21 March 2004, under the auspices of ESMO. Ann Oncol 2005, 16(4), 566-578.

16. Trent J. A prospective, randomized, phase II study of preoperative plus postoperative imatinib mesylate (Gleevec, formerly STI-571) in patients with primary, recurrent or metastatic resectable kitexpressing, gastrointestinal tumor (GIST). Available from: http:// utmext01a.mdacc.tmc.edu/dept/prot/clinicaltrialswp.nsf/index/ ID03-0023.; 2004. 